\title{
q-FREQUENT HYPERCYCLICITY IN SPACES OF OPERATORS
}

\author{
MANJUL GUPTA AND ANEESH MUNDAYADAN
}

\begin{abstract}
We provide conditions for a linear map of the form $C_{R, T}(S)=R S T$ to be $q$-frequently hypercyclic on algebras of operators on separable Banach spaces. In particular, if $R$ is a bounded operator satisfying the $q$-Frequent Hypercyclicity Criterion, then the map $C_{R}(S)=R S R^{*}$ is shown to be $q$-frequently hypercyclic on the space $\mathcal{K}(H)$ of all compact operators and the real topological vector space $\mathcal{S}(H)$ of all self-adjoint operators on a separable Hilbert space $H$. Further we provide a condition for $C_{R, T}$ to be $q$-frequently hypercyclic on the Schatten von Neumann classes $S_{p}(H)$. We also characterize frequent hypercyclicity of $C_{M_{\varphi}^{*}, M_{\psi}}$ on the trace-class of the Hardy space, where the symbol $M_{\varphi}$ denotes the multiplication operator associated to $\varphi$.
\end{abstract}

\section{INTRODUCTION}

This paper is a continuation of our earlier work [14] on $q$-frequent hypercyclicity, which coincides with frequent hypercyclicity for $q=1$. We study here this concept for linear maps defined on Banach algebras of operators on Banach and Hilbert spaces. Hypercyclicity in spaces of operators was initiated by K.C. Chan [7] and subsequently studied by J. Bonet, F. Martinez-Gimenez and A. Peris [3], K.C. Chan and R. Taylor [8], F. Martinez-Gimenez and A. Peris [17] and H. Petersson [19]. Indeed, left multiplication operators $\mathfrak{L}_{R}(S)=R S$ were considered in [3], [7], [8] and [17] and their general form $C_{R, T}(S)=R S T$ was studied in [19]. A collective work in [3], [7] and [8] states that a bounded operator $R$ on a separable Banach space $X$ satisfies the Hypercyclicity Criterion if and only if the left multiplication operator $\mathfrak{L}_{R}$ is hypercyclic on $\mathcal{L}(X)$ in the topology of pointwise convergence. This result holds for the topology of uniform convergence on compact subsets if $X^{*}$ is separable and $X$ has the approximation property, see [3]. In [19] H. Petersson proved that if $T$ satisfies the Hypercyclicity Criterion in a separable Hilbert space, then $\mathfrak{L}_{T}$ as well as the conjugate operator $C_{T}$ is hypercyclic on the Schatten von Neumann classes $S_{p}(H), 1 \leq p<\infty$ and $\mathcal{K}(H)$.

In Section 3 we provide a sufficient criterion for $C_{R, T}$ to be $q$-frequently hypercyclic on the algebra of compact operators on Banach spaces and give applications to the unilateral and bilateral shift operators, and in Section 4 we continue the study in the space $S_{p}(H)$. Finally in Section 5 , using an Eigenvalue Criterion, we characterize frequent hypercyclicity of certain maps of the form $C_{R, T}$ defined on spaces of operators on the classical Hardy space and $\ell^{p}$.

Date: 19.02 .2016 .

2010 Mathematics Subject Classification. Primary 47A16; Secondary 46A45.

Key words and phrases. unilateral shift; unconditional convergence; frequently hypercyclic; multiplication operator; Hardy space. 


\section{Preliminaries}

A continuous operator $T$ on a topological vector space (TVS) $X$ is said to be hypercyclic if the set $\left\{T^{n} x: n \geq 1\right\}$ is dense in $X$ for some $x \in X$. For $q \in \mathbb{N}$ (the set of natural numbers), $T$ is said to be $q$-frequently hypercyclic (see [14]) if there exists a vector $x \in X$ such that the set $\left\{n \in \mathbb{N}: T^{n} x \in U\right\}$ has positive $q$-lower density for each non-empty open set $U \subset X$, where the $q$-lower density of $A \subset \mathbb{N}$ is defined as

$$
\underline{q-\operatorname{dens}}(A)=\liminf _{N \rightarrow \infty} \frac{\operatorname{card}\left\{n \in A: n \leq N^{q}\right\}}{N} .
$$

For $q=1$, the above notion is known as frequent hypercyclicity of an operator, studied in [1, 4] and [5]. If $T$ is frequently hypercyclic, then it is $q$-frequently hypercyclic for all $q \in \mathbb{N}$, however, the converse is not true, cf. [14].

Let $X$ and $Y$ be separable Banach spaces. The space of all bounded (resp. of all compact) operators from $X$ to $Y$ is denoted by $\mathcal{L}(X, Y)$ (resp. $\mathcal{K}(X, Y)$ ). We shall use the symbols $\mathcal{L}(X)$ and $\mathcal{K}(X)$ for $\mathcal{L}(X, X)$ and $\mathcal{K}(X, X)$ respectively. The real subspace of $\mathcal{L}(H)$, of all self-adjoint operators on a separable infinite dimensional Hilbert space $H$ is denoted by $\mathcal{S}(H)$ and is equipped with the topology of uniform convergence on compact subsets (COT). Also, for $p \in[1, \infty)$ the Schatten von Neumann class $S_{p}(H)$ is defined as the space of all operators $T \in \mathcal{L}(H)$ for which the approximation numbers $\left(a_{n}(T)\right) \in \ell^{p}$, where

$$
a_{n}(T)=\inf \{\|T-F\|: \operatorname{rank}(F)<n\}, n \geq 1,
$$

See [10] and [11] for more details on the Schatten von Neumann classes.

For $R \in \mathcal{L}(X)$, the left and right multiplication operators are respectively defined as $\mathfrak{L}_{R}(S)=$ $R S$ and $\mathfrak{R}_{R}(S)=S R$. Also, if $R$ is a bounded operator on a Hilbert space, then the conjugate operator $C_{R}$ is defined as $C_{R}(S)=R S R^{*}$.

Recall that a Banach space $X$ is said to have the approximation property (AP) if the identity operator on $X$ can be approximated by finite rank operators uniformly on compact subsets of $X$; that is, for any $\epsilon>0$ and $K \subset X$ compact, there exists an operator $F$ of finite rank such that $\|F(x)-x\|<\epsilon, \forall x \in K$. If $X$ has the $\mathrm{AP}$, then finite rank operators are norm-dense in $\mathcal{K}(Y, X)$ for all Banach spaces $Y$, cf. [10] and [16].

A series $\sum_{n \geq 1} x_{n, j}$ in an $F$-space is said to be unconditionally convergent uniformly in $j \geq 0$ if for every $\delta>0$, there exists $N \in \mathbb{N}$ such that $\left\|\sum_{n \in F} x_{n, j}\right\|<\delta$ for all finite sets $F \subset[N, \infty)$ and $j$. We will make use of the following inequality in our subsequent work: let $\left(\lambda_{n}\right)$ be a scalar sequence and $\sum_{n \geq 1} x_{n}$ a series in a Banach space. Then if $F \subset \mathbb{N}$ is finite, we have

$$
\left\|\sum_{n \in F} \lambda_{n} x_{n}\right\| \leq 4 \sup _{n \in F}\left|\lambda_{n}\right| \sup _{G \subseteq F}\left\|\sum_{n \in G} x_{n}\right\|,
$$

cf. [18] (See also [15]). 


\section{3. $q$-Frequent HypercyClicity in $\mathcal{K}(X), \mathcal{L}(X)$ AND $\mathcal{S}(H)$}

In this section, we first obtain a sufficient criterion for the $q$-frequent hypercyclicity of $C_{R, T}$ on the Banach algebras $\mathcal{L}(X, Y)$ and $\mathcal{K}(X, Y)$, where $R \in \mathcal{L}(Y)$ and $T \in \mathcal{L}(X)$. The next result is already known for $q=1$, cf. [13], Remark 9.10. However, following the proof of the frequent hypercyclicity criterion given in Theorem 6.18 of [2], we outline the proof for a given $q \in \mathbb{N}$.

Theorem 3.1. (q-FHC Criterion) Let $X$ be a separable $F$-space and $D$ be a dense set in $X$. If for each $x \in D$, there exists a sequence $\left(x_{n}\right)_{n \geq 0}$ in $X$ such that $x_{0}=x$ and

(a) $\sum_{n=0}^{r} T^{r^{q}-(r-n)^{q}}(x)$ converges unconditionally, uniformly in $r \geq 0$,

(b) $\sum_{n \geq 0} x_{(n+r)^{q}-r^{q}}$ converges unconditionally, uniformly in $r \geq 0$; and

(c) $T^{n^{q}} x_{n^{q}}=x, T^{n^{q}} x_{m^{q}}=x_{m^{q}-n^{q}}$ for $m>n \geq 0$,

then $T$ is q-frequently hypercyclic on $X$.

Proof. Without loss of generality, one may assume that $D$ is countable. Write $D=\left\{x_{k}: k \in \mathbb{N}\right\}$ and fix $\left(\epsilon_{k}\right)$ such that $k \epsilon_{k}+\sum_{j \geq k+1} \epsilon_{j} \rightarrow 0$. For each $x_{k}$, there exists a sequence $\left(x_{n, k}\right)_{n \geq 0}$ with the conditions in the hypotheses being satisfied. By (a) and (b), it is possible to find an increasing sequence $\left(N_{k}\right)$ of natural numbers such that $\left\|\sum_{n \in F} T^{r^{q}-(r-n)^{q}}\left(x_{i}\right)\right\|<\epsilon_{k}$ for $F \subset\left[N_{k}, \infty\right) \cap\{1, \ldots, r\}$, and $\left\|\sum_{n \in G} x_{(n+r)^{q}-r^{q}, i}\right\|<\epsilon_{k}$ for $G \subset\left[N_{k}, \infty\right)$, uniformly in $r \geq 0$, where $1 \leq i \leq k$. By Lemma 6.19 of [2], corresponding to $\left(N_{k}\right)$, we find a sequence $\left(J_{k}\right)$ of subsets of $\mathbb{N}$ such that $\underline{\text { dens }}\left(J_{k}\right)>0$, $\min \left(J_{k}\right) \geq k$ and $|m-n| \geq N_{k}+N_{j}$ for all $m \in J_{k}, n \in J_{j}$ and $m \neq n$. With these properties, the vector $x=\sum_{\ell \geq 1} \sum_{n \in J_{\ell}} x_{n^{q}, \ell}$ is a frequently hypercyclic vector for the sequence $\left(T^{n^{q}}\right)$, and thus it is a $q$-frequently hypercyclic vector for $T$.

Let $y \otimes x^{*}$ be the one-rank operator $x \rightarrow x^{*}(x) y$, where $y \in Y$ and $x^{*} \in X^{*}$ and $\left(\mathcal{I}(X, Y),\|\cdot\|_{\mathcal{I}(X, Y)}\right)$ be a Banach space of operators from $X$ to $Y$ such that the set of all finite-rank operators is $\|\cdot\|_{\mathcal{I}(X, Y)^{-}}$ dense in $\mathcal{I}(X, Y)$ and $\left\|y \otimes x^{*}\right\|_{\mathcal{I}(X, Y)}=\|y\|\left\|x^{*}\right\|$ for all $y \in Y$ and $x^{*} \in X^{*}$. We have the following result concerning the separability of $\mathcal{L}(X, Y)$ with respect to the topologies SOT and COT, and of $\mathcal{K}(X, Y)$ in the operator norm topology.

Proposition 3.2. Let $X$ and $Y$ be separable Banach spaces. Then the following are true. (1) If $D$ is a countable dense subset of $Y$ and $\Phi$ is a countable weak $k^{*}$ dense subset of $X^{*}$, then the set

$$
\mathcal{G}_{D, \Phi}=\left\{\sum_{n=1}^{N} y_{n} \otimes x_{n}^{*}: y_{n} \in D, x_{n}^{*} \in \Phi, N \in \mathbb{N}\right\}
$$

is a countable SOT-dense subset of $\mathcal{L}(X, Y)$.

(2) If $X^{*}$ is separable and $\Phi$ is norm-dense, then the above set $\mathcal{G}_{D, \Phi}$ is $\|\cdot\|_{\mathcal{I}(X, Y)^{-}}$dense in $\mathcal{I}(X, Y)$.

(3) Suppose that $X^{*}$ is separable and $Y$ has the AP. If $\Phi$ is norm-dense, then $\mathcal{G}_{D, \Phi}$ is norm-dense in $\mathcal{K}(X, Y)$ and $C O T$-dense in $\mathcal{L}(X, Y)$.

Proof. The proof of (1) is similar to the case of $X=Y$, proved in [13, p. 277. Further, by the properties of $\mathcal{I}(X, Y)$ mentioned above, part (2) follows since we can approximate every operator of 
finite rank by elements of $\mathcal{G}_{D, \Phi}$ in the norm $\|\cdot\|_{\mathcal{I}(X, Y)}$. To get part (3), let us assume that $Y$ has the AP. Then the space $\mathcal{F}(X, Y)$ of all finite-rank operators is norm-dense in $\mathcal{K}(X, Y)$ for every Banach space $X$. Moreover, $\left\|v \otimes u^{*}\right\|_{o p}=\|v\|\left\|u^{*}\right\|$ for all $v \in Y$ and $u^{*} \in X^{*}$.

Using the above proposition, we prove

Theorem 3.3. Let $R \in \mathcal{L}(Y)$ and $T \in \mathcal{L}(X)$ for separable Banach spaces $X$ and $Y$ and $q \in \mathbb{N}$. Let $\mathcal{D}$ be a norm-dense set in $Y$ and $\Phi$ be a countable weak ${ }^{*}$-dense set in $X^{*}$. Suppose that for each $\left(y, x^{*}\right) \in \mathcal{D} \times \Phi$, there exist sequences $\left(y_{n}\right)_{n \geq 0}$ in $Y$ and $\left(x_{n}^{*}\right)_{n \geq 0}$ in $X^{*}$ such that

(a) the series $\sum_{n=0}^{r} R^{r^{q}-(r-n)^{q}}(y) \otimes\left(T^{*}\right)^{r^{q}-(r-n)^{q}}\left(x^{*}\right)$ and $\sum_{n=1}^{\infty} y_{(n+r)^{q}-r^{q}} \otimes x_{(n+r)^{q}-r^{q}}^{*}$ are unconditionally convergent in $\left(\mathcal{L}(X, Y),\|\cdot\|_{\text {op }}\right)$, uniformly in $r \geq 0$; and

(b) $R^{n^{q}} y_{n^{q}}=y,\left(T^{*}\right)^{n^{q}} x_{n^{q}}^{*}=x^{*}, R^{n^{q}} y_{m^{q}}=y_{m^{q}-n^{q}}$, and $\left(T^{*}\right)^{n^{q}} x_{m^{q}}^{*}=x_{m^{q}-n^{q}}^{*}$ for all $m>n \geq 0$.

Then the following assertions hold.

(i) If $T^{*}(\Phi) \subseteq \Phi,\left\{x_{n}^{*}\right\} \subseteq \Phi$, then $C_{R, T}$ is q-frequently hypercyclic on $(\mathcal{L}(X, Y)$, SOT).

(ii) If $Y$ has the $A P$ and the set $\Phi$ is norm-dense in $X^{*}$, then $C_{R, T}$ is q-frequently hypercyclic on $\left(\mathcal{K}(X, Y),\|\cdot\|_{\text {op }}\right)$ and $(\mathcal{L}(X, Y), \mathrm{COT})$.

Proof. (i) As $Y$ is a separable Banach space, we may assume that $D$ is countable. Let

$$
\mathcal{L}_{\Phi}=\overline{\operatorname{span}}^{\|\cdot\|_{o p}}\left\{y \otimes x^{*}: y \in Y, x^{*} \in \Phi\right\}
$$

where the closure is taken in the operator norm $\|\cdot\|_{o p}$. Then $\mathcal{L}_{\Phi}$ is a separable Banach space since the set

$$
\mathcal{G}_{D, \Phi}=\left\{\sum_{n=1}^{N} y_{n} \otimes x_{n}^{*}: y_{n} \in D, x_{n}^{*} \in \Phi, N \in \mathbb{N}\right\}
$$

is countable and norm-dense in $\mathcal{L}_{\Phi}$.

If $G=\sum_{j \leq N} y_{j} \otimes x_{k}^{*}$, then $C_{R, T}(G)=\sum_{j \leq N} R\left(y_{j}\right) \otimes T^{*}\left(x_{j}^{*}\right)$. As the map $C_{R, T}$ is continuous and $T^{*}(\Phi) \subseteq \Phi$, it follows that $C_{R, T}$ takes $\mathcal{L}_{\Phi}$ to itself.

To establish the $q$-frequent hypercyclicity of the operator $C_{R, T}$ in SOT, we first show that $C_{R, T}$ satisfies the conditions of Theorem 3.1 in the space $\mathcal{L}_{\Phi}$. Let $F=\sum_{j=1}^{k} y_{j} \otimes x_{j}^{*} \in \mathcal{G}_{\mathcal{D}, \Phi}$. For each $\left(y_{j}, x_{j}^{*}\right)$, let $\left(y_{j, n}\right)$ and $\left(x_{j, n}^{*}\right)$ be some sequences, respectively in $Y$ and $\Phi$, as in the hypothesis. Then

$$
F_{n}=\sum_{j \leq k} y_{j, n} \otimes x_{j, n}^{*} \in \mathcal{L}_{\Phi}, n \geq 0
$$

Therefore, by the assumption (a) of our theorem, both the series $\sum_{n \leq r}\left(C_{R, T}\right)^{r^{q}-(r-n)^{q}}(F)=\sum_{j \leq k} \sum_{n \leq r} R^{r^{q}-(r-n)^{q}}\left(y_{j}\right) \otimes\left(T^{*}\right)^{r^{q}-(r-n)^{q}}\left(x_{j}^{*}\right)$ and $\sum_{n \geq 0} F_{(n+r)^{q}-r^{q}}=$ $\sum_{j \leq k} \sum_{n \geq 0} y_{j,(n+r)^{q}-r^{q}} \otimes x_{j,(n+r)^{q}-r^{q}}^{*}$ converge unconditionally in $\mathcal{L}_{\Phi}$ with respect to the operator norm, uniformly in $r \geq 0$. 
Further, we have

$$
\begin{aligned}
\left(C_{R, T}\right)^{n^{q}} F_{m^{q}} & =\sum_{j=1}^{k} R^{n^{q}} y_{j, m^{q}} \otimes\left(T^{*}\right)^{n^{q}} x_{j, m^{q}}^{*} \\
& =\left\{\begin{array}{l}
\sum_{j=1}^{k} y_{j, m^{q}-n^{q}} \otimes x_{j, m^{q}-n^{q}}^{*}, m>n \\
F, m=n .
\end{array}\right.
\end{aligned}
$$

by hypotheses. Thus $\left(C_{R, T}\right)^{n^{q}} F_{m^{q}}=F_{m^{q}-n^{q}}$ if $m>n$ and $\left(C_{R, T}\right)^{n^{q}} F_{n^{q}}=F, n \geq 0$. So $C_{R, T}$ is $q$-frequently hypercyclic on $\mathcal{L}_{\Phi}$ with respect to the operator norm topology. As $\mathcal{G}_{D, \Phi}$ is SOT-dense in $\mathcal{L}(X, Y)$ by Proposition 3.2 , the operator $C_{R, T}$ is $q$-frequently hypercyclic on $(\mathcal{L}(X, Y), S O T)$. This establishes part (i).

Let us now prove part (ii). If $Y$ has the AP and the set $\Phi$ is norm-dense in $X^{*}$, then by Proposition $3.2(3), \mathcal{G}_{D, \Phi}$ is dense in $\mathcal{K}(X, Y)$ with respect to the operator norm and so $\mathcal{L}_{\Phi}=\mathcal{K}(X, Y)$. Consequently, $C_{R, T}$ is $q$-frequently hypercyclic on $\left(\mathcal{K}(X, Y),\|\cdot\|_{\text {op }}\right)$ and $(\mathcal{L}(X, Y), \mathrm{COT})$.

For applications of Theorem 3.3 , we require the following lemmas.

Lemma 3.4. Let $X$ and $Y$ be Banach spaces. If $\sum_{n=1}^{\infty} u_{n, j}$ is unconditionally convergent in $Y$, uniformly in $j \geq 0$, and $\left\{u_{n, k}^{*}\right\} \subset X^{*}$ is such that $\left\{u_{n, k}^{*}: n \geq N_{0}, k \geq 1\right\}$ is norm-bounded for some $N_{0} \in \mathbb{N}$, then $\sum_{n} u_{n, j} \otimes u_{n, k}^{*}$ is unconditionally convergent in $\left(\mathcal{L}(X, Y),\|\cdot\|_{\text {op }}\right)$, uniformly in $j, k \in \mathbb{N}$.

Proof. By definition, for a given $\epsilon>0$, one can choose a natural number $N>N_{0}$ such that $\left\|\sum_{n \in F} u_{n, j}\right\|<\epsilon$ for every finite set set $F \subset[N, \infty) \cap \mathbb{N}$ and all $j \geq 1$.

Let $M \in \mathbb{R}$ be such that $\left\|u_{n, k}^{*}\right\| \leq M \forall n \geq N_{0}, k \geq 1$. Using the inequality (2.1), it follows that

$$
\left\|\sum_{n \in F} u_{n, k}^{*}(x) u_{n, j}\right\| \leq 4 M \sup _{G \subseteq F}\left\|\sum_{n \in G} u_{n, j}\right\|<4 M \epsilon,
$$

for $j, k \geq 1$ and $\|x\| \leq 1$. Thus

$$
\left\|\sum_{n \in F} u_{n, j} \otimes u_{n, k}^{*}\right\|_{o p}<4 M \epsilon
$$

Lemma 3.5. Let $X$ and $Y$ be Banach spaces. If $\sum_{n=1}^{j} u_{n, j}$ is unconditionally convergent in $Y$, uniformly in $j \in \mathbb{N}$, and $\left\{u_{n, j}^{*}: n \geq N_{0}, k \geq 1\right\}$ is norm-bounded in $X^{*}$ for some $N_{0} \in \mathbb{N}$, then the series $\sum_{n}^{j} u_{n, j} \otimes u_{n, j}^{*}$ is unconditionally convergent in $\left(\mathcal{L}(X, Y),\|\cdot\|_{o p}\right)$, uniformly in $j \in \mathbb{N}$.

Recalling the $q$-FHC Criterion from Theorem 3.1, we prove the $q$-frequent hypercyclicity of the left multiplication operator $\mathfrak{L}_{R}(S)=R S$. This strengthens a result of A. Bonilla and K.-G. Grosse-Erdmann [5] about the SOT-frequent hypercyclicity of $\mathfrak{L}_{R}$. 
Corollary 3.6. Let $X$ be a separable Banach space and $R \in \mathcal{L}(X)$ satisfy the q-FHC Criterion. Then the following hold.

(i) $\mathfrak{L}_{R}$ is q-frequently hypercyclic on $(\mathcal{L}(X), S O T)$.

(ii) If $X^{*}$ is separable and $X$ has the $A P$, then the $\mathfrak{L}_{R}$ is q-frequently hypercyclic on $\left(\mathcal{K}(X),\|\cdot\|_{o p}\right)$ and $(\mathcal{L}(X), C O T)$.

Proof. In Theorem 3.3, let $X=Y$ and $T$ be the identity operator on $X$. Since $X$ is separable, the dual $X^{*}$ is weak ${ }^{*}$-separable. So, we can choose $\Phi$ to be any countable weak*-dense subset of $X^{*}$. Since $R$ satisfies the $q$-FHC Criterion, we find a set $D$ satisfying the conditions (a) and (b) of Theorem 3.1. That is, for $x \in D$, there exists $\left(x_{n}\right)$ in $X$ with $x_{0}=x$ such that

$$
\sum_{n \leq r} R^{r^{q}-(r-n)^{q}}(x) \text { and } \sum_{n \geq 1} x_{(n+r)^{q}-r^{q}} \text { are unconditionally convergent uniformly in } r \geq 0,
$$

and

$$
R^{n^{q}} x_{n^{q}}=x, R^{n^{q}} x_{m^{q}}=x_{m^{q}-n^{q}}, m>n, n \geq 0 .
$$

Now we verify the condition $(a)$ of Theorem 3.3. For each $x^{*} \in \Phi$, let $x_{n}^{*}=x, n \geq 0$. By Lemmas 3.4 and 3.5 we get (i).

To see (ii), take $\Phi$ as any norm-dense subset of $X^{*}$ in Theorem 3.3 and apply Theorem 3.3(ii).

Similarly, one can prove the following results. We observe that if $\sum_{n} x_{n, j}$ is unconditionally convergent, uniformly in $j$, then there exists $N \in \mathbb{N}$ such that the set $\left\{x_{n, j}: n \geq N, j \geq 1\right\}$ is bounded.

Corollary 3.7. Suppose $X$ is a separable Banach space and $T \in \mathcal{L}(X)$. Then the following are true. (1) Let $\Phi$ be a countable weak $k^{*}$-dense subset of $X^{*}$. Suppose that for each $x^{*} \in \Phi$, there exists $\left(x_{n}^{*}\right)$ in $\Phi$ with properties that $x_{0}^{*}-x^{*}$, the series $\sum_{n \leq r}\left(T^{*}\right)^{r^{q}-(r-n)^{q}}\left(x^{*}\right)$ and $\sum_{n \geq 1} x_{(n+r)^{q}-r^{q}}^{*}$ are unconditionally convergent in $\left(X^{*},\|\|.\right)$, uniformly in $r$; and $\left(T^{*}\right)^{n^{q}} x_{n^{q}}^{*}=x^{*},\left(T^{*}\right)^{n^{q}} x_{m^{q}}^{*}=x_{m^{q}-n^{q}}^{*}$, $m>n$. If $T^{*}(\Phi) \subset \Phi$, then $\mathfrak{R}_{T}$ is q-frequently hypercyclic on $(\mathcal{L}(X), S O T)$.

(2) Assume that $X^{*}$ is separable and $X$ has the AP. If $T^{*}$ satisfies the $q$-FHC Criterion, then $\mathfrak{R}_{T}$ is q-frequently hypercyclic on $\left(\mathcal{K}(X),\|.\|_{\text {op }}\right)$ and $(\mathcal{L}(X), C O T)$.

Proposition 3.8. Let $R, T \in \mathcal{L}(X)$, where $R$ satisfies the $q-F H C$ Criterion, and let $\Phi$ be a countable weak ${ }^{*}$-dense set in $X^{*}$. If for each $f \in \Phi$, there exists a bounded $\left(f_{n}\right)$ in $X^{*}$ such that $f_{0}=0$ and the set $\left\{\left(T^{*}\right)^{n}(f): n \geq 0\right\}$ is bounded; and $\left(T^{*}\right)^{n^{q}} f_{n^{q}}=f,\left(T^{*}\right)^{n^{q}} f_{m^{q}}=f_{m^{q}-n^{q}}$ for $m>n \geq 0$, then the following hold.

(1) If $T^{*}(\Phi) \subseteq \Phi$, then $C_{R, T}$ is q-frequently hypercyclic on $(\mathcal{L}(X), S O T)$.

(2) If $\Phi$ is norm-dense, and $X$ has the $A P$, then $C_{R, T}$ is q-frequently hypercyclic on $\left(\mathcal{K}(X),\|\cdot\|_{o p}\right)$ and $(\mathcal{L}(X), C O T)$.

Let us now establish the $q$-frequent hypercyclicity of $C_{R, U}$ for a unitary $U$ and of the conjugate operator $C_{R}(S)=R S R^{*}$. 
Corollary 3.9. Let $R$ satisfy the $q-F H C$ Criterion in a separable Hilbert space $H$. Then the operators $C_{R}$ and $C_{R, U}$ are q-frequently hypercyclic on $\left(\mathcal{K}(H),\|\cdot\|_{\text {op }}\right)$ and $(\mathcal{L}(H), C O T)$, where $U \in \mathcal{L}(H)$ is a unitary operator.

Proof. Since $H$ is a separable Hilbert space, it has the AP. As in the proof of the above results, one can find a dense set $D$ of $H$ satisfying the conditions in Theorem 3.1 . Now, in Proposition 3.8 take $T=R^{*}$ and $\Phi=D$. The result follows.

We now proceed to some concrete applications of Theorem 3.3. We provide sufficient conditions on the weights $\left(w_{n}\right)$ and $\left(\mu_{n}\right)$ for the map $C_{B_{w}, F_{\mu}}$ to be $q$-frequently hypercyclic on different Banach algebras of operators on $\ell^{p}, 1 \leq p<\infty$, where the backward shift $B_{w}$ and the forward shift $F_{\mu}$ are respectively given by $B_{w}\left(e_{0}\right)=0, B_{w}\left(e_{n}\right)=w_{n} e_{n-1}, n \geq 1$ and $F_{\mu}\left(e_{n}\right)=\mu_{n+1} e_{n+1}, n \geq 0$. Here $\left\{e_{n}\right\}_{n \geq 0}$ is the standard basis in $\ell^{p}$.

Proposition 3.10. If $\lim _{n \rightarrow \infty}\left|w_{1} w_{2} . . w_{(n+r)^{q}-r^{q}+i} \mu_{1} \mu_{2} . . \mu_{(n+r)^{q}-r^{q}+j}\right|=\infty$, uniformly in $r \geq 0$, for all $i, j \in \mathbb{N}_{0}$, then $C_{B_{w}, F_{\mu}}$ is q-frequently hypercyclic on $\left(\mathcal{L}\left(\ell^{1}\right), \operatorname{SOT}\right),\left(\mathcal{K}\left(\ell^{p}\right),\|\cdot\|_{o p}\right)$ and $\left(\mathcal{L}\left(\ell^{p}\right), \mathrm{COT}\right)$, where $1<p<\infty$.

Proof. In Theorem 3.3, let $X=Y=\ell^{p}$. To prove the result for $\left(\mathcal{L}\left(\ell^{1}\right), \mathrm{SOT}\right)$, let us write $\Phi_{0}$ for the linear span of $\left\{e_{n}^{*}: n \geq 0\right\}$ over rationals in $\ell^{\infty}$ and $\mathcal{D}$ for $\operatorname{span}\left\{e_{n}: n \geq 0\right\}$ in $\ell^{1}$. Consider the maps $S_{w}$ and $J_{\mu}$ given by $S_{w}\left(e_{n}\right)=\frac{1}{w_{n+1}} e_{n+1}$ and $J_{\mu}\left(e_{n}^{*}\right)=\frac{1}{\mu_{n+1}} e_{n+1}^{*}, n \geq 0$. Note that $B_{w} S_{w}$ and $F_{\mu}^{*} J_{\mu}$ are identity operators, and

$$
S_{w}^{m}\left(e_{n}\right)=\frac{1}{w_{n+1} w_{n+2} \ldots w_{n+m}} e_{n+m} \text { and } J_{\mu}^{m}\left(e_{n}^{*}\right)=\frac{1}{\mu_{n+1} \mu_{n+2} \ldots \mu_{n+m}} e_{n+m}^{*} .
$$

Put

$$
\Phi=\bigcup_{j \geq 0} \Phi_{j}, \text { where } \Phi_{j+1}=\bigcup_{n, k \geq 0}\left(F_{\mu}^{*}\right)^{n} J_{\mu}^{k}\left(\Phi_{j}\right), j \geq 0 .
$$

Then the set $\Phi$ is weak ${ }^{*}$-dense in $\ell^{\infty}$ as $\Phi_{0} \subseteq \Phi$. Clearly $\Phi$ is countable. Further $F_{\mu}^{*}(\Phi) \subseteq \Phi$ and $J_{\mu}(\Phi) \subseteq \Phi$.

We only consider the series

$$
\sum_{n=1}^{r} B_{w}^{r^{q}-(r-n)^{q}}\left(e_{i}\right) \otimes\left(F_{\mu}^{*}\right)^{r^{q}-(r-n)^{q}}\left(e_{j}^{*}\right) \text { and } \sum_{n=1}^{\infty} S_{w}^{(n+r)^{q}-r^{q}}\left(e_{i}\right) \otimes J_{\mu}^{(n+r)^{q}-r^{q}}\left(e_{j}^{*}\right)
$$

for $i, j \geq 0$. As $B_{w}^{n}\left(e_{i}\right)=0$ for sufficiently large $n$ and $r^{q}-(r-n)^{q} \geq n$, the first series converges unconditionally in the operator norm, uniformly in $r \geq 0$. For the latter series, it suffices to prove that $\sum_{n \geq 1} a_{n, r} e_{(n+r)^{q}-r^{q}+i} \otimes e_{(n+r)^{q}-r^{q}+j}^{*}$ converges unconditionally, uniformly in the operator norm if $\lim _{n \rightarrow \infty}\left|a_{n, r}\right|=0$, uniformly in $r$. But this is immediate as, for $x=\left(x_{n}\right) \in \ell^{p}, 1 \leq p<\infty$, we have

$$
\begin{gathered}
\left\|\sum_{n \in F} a_{n, r} e_{(n+r)^{q}-r^{q}+i} \otimes e_{(n+r)^{q}-r^{q}+j}^{*}\right\|=\left\|\sum_{n \in F} a_{n, r} x_{(n+r)^{q}-r^{q}+j} e_{(n+r)^{q}-r^{q}+i}\right\|= \\
\left(\sum_{n \in F}\left|a_{n, r} x_{(n+r)^{q}-r^{q}+j}\right|^{p}\right)^{1 / p} \leq \max _{n \in F}\left|a_{n, r}\right|\|x\| .
\end{gathered}
$$


Therefore $C_{B_{w}, F_{\mu}}$ is $q$-frequently hypercyclic on $\left(\mathcal{L}\left(\ell^{1}\right), S O T\right)$ by Theorem 3.3 (i).

Since $\ell^{p}$ has the AP and the set $\Phi$ constructed above is norm-dense in $\ell^{p^{\prime}}$, where $1<p<$ $\infty, 1 / p+1 / p^{\prime}=1$ and $\ell^{p^{\prime}}$ is the dual of $\ell^{p}$, the operator $C_{B_{w}, F_{\mu}}$ is $q$-frequently hypercyclic on $\left(\mathcal{K}\left(\ell^{p}\right),\|\cdot\|_{o p}\right)$ and $\left(\mathcal{L}\left(\ell^{p}\right), C O T\right)$ by Theorem $[3.3($ ii $)$.

Remark 1. It is evident from the proof of the above proposition that the result holds for any Banach sequence space $E$ with the AP such that $\operatorname{span}\left\{e_{n}: n \geq 0\right\}$, span $\left\{e_{n}^{*}\right\}$ over rationals are norm-dense in $E, E^{*}$ respectively and $\sum_{n}\left(w_{1} w_{2} . . w_{i+(n+r)^{q}-r^{q}} \mu_{1} \mu_{2} . . \mu_{j+(n+r)^{q}-r^{q}}\right)^{-1} e_{i+(n+r)^{q}-r^{q}} \otimes e_{j+(n+r)^{q}-r^{q}}^{*}$ converges unconditionally in the operator norm, uniformly in $r$ for all $i, j \geq 0$.

Our next aim is to obtain the bilateral version of Proposition 3.10. For $a=\left(a_{n}\right)_{n \in \mathbb{Z}}$ of a bounded sequence of nonzero scalars, we define the bilateral backward shift $T_{a}$ on the sequence space $\ell^{p}(\mathbb{Z}), 1 \leq p<\infty$, as $T_{a}\left(e_{n}\right)=a_{n} e_{n-1}$ and the forward shift $S_{a}$ as $S_{a}\left(e_{n}\right)=a_{n} e_{n+1}, n \in \mathbb{Z}$, where $\left\{e_{n}\right\}_{n \in \mathbb{Z}}$ is the standard basis in $\ell^{p}(\mathbb{Z})$. Then we have

Proposition 3.11. Suppose that, for all $i, j \in \mathbb{Z}, \lim _{n \rightarrow \infty}\left|a_{1} a_{2} . . a_{(n+r)^{q}-r^{q}+i} b_{1} b_{2} . . b_{(n+r)^{q}-r^{q}+j}\right|=$ $\infty$ and $\lim _{n \rightarrow \infty}\left|a_{i} a_{i-1} . . a_{i-r^{q}+(r-n)^{q}+1} b_{j} b_{j-1} . . b_{j-r^{q}+(r-n)^{q}+1}\right|=0$, uniformly in $r \in \mathbb{N}_{0}$. Then $C_{T_{a}, S_{b}}$ is q-frequently hypercyclic on $\left(\mathcal{L}\left(\ell^{1}(\mathbb{Z})\right), S O T\right),\left(\mathcal{K}\left(\ell^{p}(\mathbb{Z}),\|\cdot\|_{\text {op }}\right)\right)$ and $\left(\mathcal{L}\left(\ell^{p}(\mathbb{Z})\right), C O T\right), 1<p<\infty$.

Proof. We apply Theorem 3.3. Choose $X=Y=\ell^{p}(\mathbb{Z}), \mathcal{D}=\operatorname{span}\left\{e_{n}: n \in \mathbb{Z}\right\}$ and $\Phi_{0}=\operatorname{span}\left\{e_{n}^{*}\right.$ : $n \in \mathbb{Z}\}$ over rationals. Define the maps $S$ and $J$ as $S\left(e_{n}\right)=\frac{1}{a_{n+1}} e_{n+1}$ and $J\left(e_{n}^{*}\right)=\frac{1}{b_{n+1}} e_{n+1}^{*}$ for $n \in \mathbb{Z}$. Let

$$
\Phi=\bigcup_{j \geq 0} \Phi_{j}, \text { where } \Phi_{j+1}=\bigcup_{n, k \geq 0} S_{b}^{* n} J^{k}\left(\Phi_{j}\right), j \geq 0
$$

The set $\mathcal{D}$ is norm-dense in $\ell^{p}(\mathbb{Z})$ for $p \in[1, \infty), \Phi$ is weak ${ }^{*}$-dense in $\ell^{\infty}(\mathbb{Z})$ and norm-dense in $\ell^{p^{\prime}}(\mathbb{Z})$, where $1 / p+1 / p^{\prime}=1$ and $1<p<\infty$. Moreover $T_{a} S$ is the identity operator on $\mathcal{D}$ and $S_{b}^{*} J$ is the identity on $\Phi$. As in Proposition 3.10, $J(\Phi) \subseteq \Phi$ and $S_{b}^{*}(\Phi) \subseteq \Phi$. Further for $n \geq 1$ and $i, j \in \mathbb{Z}$, we have

$$
\begin{gathered}
T_{a}^{n}\left(e_{i}\right)=a_{i} a_{i-1} . . a_{i-n+1} e_{i-n},\left(S_{b}^{*}\right)^{n}\left(e_{j}^{*}\right)=b_{j} b_{j-1} . . b_{j-n+1} e_{j-n}^{*}, \\
S^{n}\left(e_{i}\right)=\frac{1}{a_{i+1} a_{i+2} . . a_{i+n}} e_{i+n} \text { and } J^{n}\left(e_{j}^{*}\right)=\frac{1}{b_{j+1} b_{j+2} . . b_{j+n}} e_{j+n}^{*} .
\end{gathered}
$$

Let $S_{n}=S^{n}$ and $J_{n}=J^{n}$. Proceeding as in the proof of Proposition 3.10, one can show that the series $\sum_{n \leq r} T_{a}^{r^{q}-(r-n)^{q}}\left(e_{i}\right) \otimes\left(S_{b}^{*}\right)^{r^{q}-(r-n)^{q}}\left(e_{j}^{*}\right)$ and $\sum_{n \geq 1} S_{(n+r)^{q}-r^{q}}\left(e_{i}\right) \otimes J_{(n+r)^{q}-r^{q}}\left(e_{j}^{*}\right)$ are unconditionally convergent in the operator norm, uniformly in $r \geq 0$, by the hypothesis. This proves part (1).

Also since $\ell^{p}(\mathbb{Z})$ has the AP, and the above set $\Phi$ is norm-dense in $\ell^{p^{\prime}}(\mathbb{Z})(1<p<\infty$ and $\frac{1}{p}+\frac{1}{p^{\prime}}=1$ ), Theorem 3.3 (ii) yields $(2)$.

For the next result, let $\mathbb{C}^{N}$ be considered as a vector space over $\mathbb{C}$, and for $\lambda=\left(\lambda_{1}, \ldots, \lambda_{N}\right) \in$ $\mathbb{C}^{N}$, let $D_{\mu}$ be the diagonal operator $D_{\lambda}\left(f_{j}\right)=\lambda_{j} f_{j}$ on $\mathbb{C}^{N}$ with respect to the standard basis $\left\{f_{1}, f_{2}, . ., f_{N}\right\}$ of $\mathbb{C}^{N}$, where $N \in \mathbb{N}$. Then we have 
Proposition 3.12. Suppose that $\left|\lambda_{j}\right| \geq 1$ for each $1 \leq j \leq N$.

(1) If $\sum_{n \geq 1} \frac{1}{\left|\mu_{1} \mu_{2} \ldots \mu_{(n+r)^{q}-r^{q}+i}\right|}<\infty$ uniformly in $r$, for each $i \geq 0$, then $C_{D_{\lambda}, F_{\mu}}$ is $q$-frequently hypercyclic on $\left(\mathcal{L}\left(\ell^{1}, \mathbb{C}^{N}\right), S O T\right)$.

(2) If $1<p<\infty$ and $\sum_{n \geq 1} \frac{1}{\left|\mu_{1} \mu_{2} \ldots \mu_{(n+r)^{q}-r^{q}+i}\right|^{p}}<\infty$ uniformly in $r$, for all $i \geq 0$, then $C_{D_{\lambda}, F_{\mu}}$ is q-frequently hypercyclic on $\left(\mathcal{K}\left(\ell^{p}, \mathbb{C}^{N}\right),\|\cdot\|_{\text {op }}\right)$ and $\left(\mathcal{L}\left(\ell^{p}, \mathbb{C}^{N}\right), C O T\right)$.

Proof. In Theorem 3.3, take $X=\ell^{p}$ and $Y=\mathbb{C}^{N}$. Consider the set $\Phi$ and the maps $J_{n}$ as in the proof of Proposition 3.10. Choose $D=\mathbb{C}^{N}$ and $S_{n}=S_{\lambda}^{n}$, where $S_{\lambda}\left(f_{j}\right)=\frac{1}{\lambda_{j}} f_{j}$. For a fixed $i \geq 0$, the series $\sum_{n \leq r} D_{\lambda}^{r^{q}-(r-n)^{q}}\left(f_{j}\right) \otimes\left(F_{\mu}^{*}\right)^{r^{q}-(r-n)^{q}}\left(e_{i}\right)$ is clearly unconditionally convergent in the operator norm, uniformly in $r$. Since $S_{\lambda}^{n}\left(f_{j}\right)=\lambda_{j}^{-n} f_{j}$ and $\left|\lambda_{j}\right| \geq 1$, the set $\left\{S_{n}\left(f_{j}\right): n=0,1,2, \ldots\right\}$ becomes bounded. Consequently, the series $\sum_{n \geq 1} S_{(n+r)^{q}-r^{q}}\left(f_{j}\right) \otimes J_{(n+r)^{q}-r^{q}}\left(e_{i}\right)$ converges unconditionally, uniformly in $r$ by Lemma 3.4 .

So far, we have considered applications of Theorem 3.3 to maps on Banach algebras of operators. Now we turn to the $q$-frequent hypercyclicity of the conjugate operator $C_{R}(S)=R S R^{*}$ defined on the real subspace $\mathcal{S}(H)$ of $\mathcal{L}(H)$, consisting of all self-adjoint operators on a separable Hilbert space $H$. H. Petersson [19] showed that if $R$ satisfies the Hypercyclicity Criterion, then $C_{R}$ is hypercyclic on the norm-closure of span $\{h \otimes h: h \in H\}$ over $\mathbb{R}$, and hence COT-hypercyclic on $\mathcal{S}(H)$. A standard application of the $q$-FHC criterion, using Lemmas 3.4 and 3.5 yield the following:

Proposition 3.13. If $R \in \mathcal{L}(H)$ satisfies the $q$-FHC Criterion, then $C_{R}$ is q-frequently hypercyclic on $(\mathcal{S}(H), \mathrm{COT})$.

\section{4. $q$-Frequent Hypercyclicity in $S_{p}(H)$}

In this section we provide a sufficient condition for $C_{R, T}$ to be $q$-frequently hypercyclic on $S_{p}(H)$ for a separable Hilbert space $H$. Let us begin with the $q$-frequent hypercyclicity of the left multiplication operator $\mathfrak{L}_{R}$ on $S_{p}(H), 1 \leq p<\infty$, which is an easy application of the $q$-FHC Criterion.

Proposition 4.1. Suppose that $R$ is an operator satisfying the q-Frequent Hypercyclicity Criterion in a Banach space $X$ with separable dual.

(1) If $\left(\mathcal{I}(X),\|\cdot\|_{\mathcal{I}}\right)$ is a separable Banach ideal in $\mathcal{L}(X)$ such that the finite rank operators on $X$ are $\|\cdot\|_{\mathcal{I}}$-dense in $\mathcal{I}(X)$, and $\left\|x \otimes x^{*}\right\|_{\mathcal{I}}=\|x\|\left\|x^{*}\right\|$ for all $x \in X$ and $x^{*} \in X^{*}$, then the left multiplication operator $\mathfrak{L}_{R}$ is q-frequently hypercyclic on $\mathcal{I}(X)$.

(2) If $X$ is a separable Hilbert space, then $\mathfrak{L}_{R}$ is frequently hypercyclic on $S_{p}(X), 1 \leq p<\infty$.

Proof. The proof of (1) is omitted. To see that (2) is true, recall that $\|u \otimes v\|_{p}=\|u\|\|v\|$ for all $u, v \in X$ and the finite rank operators on $X$ form a dense subspace of $S_{p}(X)$.

For the main theorem of this section, we state the following result on summability of a series in $S_{p}(H)$, cf. [6], p. 152 . 
Lemma 4.2. Let $\left\{T_{n}\right\}_{n=1}^{\infty} \subset \mathcal{L}(H)$ be such that $T_{n}^{*} T_{m}=T_{n} T_{m}^{*}=0$ whenever $m \neq n$. Then for $1 \leq p<\infty$

$$
\left\|\sum_{n} T_{n}\right\|_{p}^{p}=\sum_{n}\left\|T_{n}\right\|_{p}^{p}
$$

We prove:

Theorem 4.3. Let $1 \leq p<\infty, R, T \in \mathcal{L}(H)$ and $D_{1}, D_{2} \subset H$. Let $D_{1}$ and $D_{2}$ both span dense subspaces of $H$. If for each $(x, y) \in D_{1} \times D_{2}$, there exist sequences $\left(x_{n}, y_{n}\right) \in H \times H$ with $\left(x_{0}, y_{0}\right)=$ $(x, y)$ and
(a) $\sum_{n=1}^{r}\left\|R^{r^{q}-(r-n)^{q}}(x)\right\|^{p}\left\|\left(T^{*}\right)^{r^{q}-(r-n)^{q}}(y)\right\|^{p}<\infty$ and $\sum_{n=1}^{\infty}\left\|x_{(n+r)^{q}-r^{q}}\right\|^{p}\left\|y_{(n+r)^{q}-r^{q}}\right\|^{p}<\infty$ uni- formly in $r \geq 0$,
(b) $\left\langle R^{n}(x), R^{m}(x)\right\rangle=\left\langle S_{n}(x), S_{m}(x)\right\rangle=\left\langle\left(T^{*}\right)^{n}(y),\left(T^{*}\right)^{m}(y)\right\rangle$ $=\left\langle J_{n}(y), J_{m}(y)\right\rangle=0$, for $m \neq n ;$ and
(c) $R^{n^{q}} x_{n^{q}}=x,\left(T^{*}\right)^{n^{q}} y_{n^{q}}=y, R^{n^{q}} x_{m^{q}}=x_{m^{q}-n^{q}},\left(T^{*}\right)^{n^{q}} y_{m^{q}}=y_{m^{q}-n^{q}}, \forall m>n \geq 0$,

then $C_{R, T}$ is q-frequently hypercyclic on $\left(S_{p}(H),\|\cdot\|_{p}\right)$.

Proof. Let $\Delta=\operatorname{span}\left\{x \otimes y: x \in D_{1}, y \in D_{2}\right\}$. Note that $\Delta$ can also be written as the span of the set $\left\{x \otimes y: x \in \operatorname{span} D_{1}, y \in \operatorname{span} D_{2}\right\}$. Since $\operatorname{span} D_{1}$ and $\operatorname{span} D_{2}$ are dense in $H$, it can be proved that $\Delta$ is dense in $S_{p}(H), 1 \leq p<\infty$.

Let $F=\sum_{k=1}^{N} a_{k} x_{k} \otimes y_{k} \in \Delta$. Corresponding to $x_{k}$ and $y_{k}$, we obtain sequences $\left(x_{k, n}\right)$ and $\left(y_{k, n}\right)$ as in the hypothesis. Set $F_{n}=\sum_{k=1}^{N} a_{k} x_{k, n} \otimes y_{k, n}$. Consider the series $\sum_{n \leq r} C_{R, T}^{r^{q}-(r-n)^{q}}(F)=$ $\sum_{k=1}^{N} a_{k} \sum_{n=1}^{\infty} R^{r^{q}-(r-n)^{q}}\left(x_{k}\right) \otimes\left(T^{*}\right)^{r^{q}-(r-n)^{q}}\left(y_{k}\right)$ and $\sum_{n} F_{(n+r)^{q}-r^{q}}=\sum_{k=1}^{N} a_{k} \sum_{n=1}^{\infty} x_{k,(n+r)^{q}-r^{q}} \otimes$ $y_{k,(n+r)^{q}-r^{q}}$. It suffices to prove that $\sum_{n \leq r} R^{r^{q}-(r-n)^{q}}(x) \otimes\left(T^{*}\right)^{r^{q}-(r-n)^{q}}(y)$ and $\sum_{n} x_{k,(n+r)^{q}-r^{q}} \otimes$ $y_{k,(n+r)^{q}-r^{q}}$ are unconditionally convergent in $S_{p}(H)$, uniformly in $r$, for all $x \in D_{1}$ and $y \in D_{2}$.

Write $T_{n, r}=R^{r^{q}-(r-n)^{q}}(x) \otimes\left(T^{*}\right)^{r^{q}-(r-n)^{q}}(y), n \geq 1$. Then $T_{n, r}^{*}=\left(T^{*}\right)^{r^{q}-(r-n)^{q}}(y) \otimes R^{r^{q}-(r-n)^{q}}(x)$. If $\left\langle\right.$. $>$ is the inner product in $H$, then $T_{n, r}^{*}(z)=\left\langle z, R^{r^{q}-(r-n)^{q}}(x)\right\rangle\left(T^{*}\right)^{r^{q}-(r-n)^{q}}(y)$ and $T_{m, r}(z)=$ $\left\langle z,\left(T^{*}\right)^{r^{q}-(r-m)^{q}}(y)\right\rangle R^{r^{q}-(r-m)^{q}}(x)$ and so

$$
T_{n, r}^{*} T_{m, r}(z)=\left\langle z,\left(T^{*}\right)^{r^{q}-(r-m)^{q}}(y)\right\rangle\left\langle R^{r^{q}-(r-m)^{q}} x, R^{r^{q}-(r-n)^{q}} x\right\rangle\left(T^{*}\right)^{r^{q}-(r-n)^{q}}(y) .
$$

Similarly

$$
T_{n, r} T_{m, r}^{*}(z)=\left\langle z, R^{r^{q}-(r-m)^{q}} x\right\rangle\left\langle\left(T^{*}\right)^{r^{q}-(r-m)^{q}} y,\left(T^{*}\right)^{r^{q}-(r-n)^{q}} y\right\rangle R^{r^{q}-(r-n)^{q}} x .
$$

From part (b) in the hypotheses, we get $T_{n, r}^{*} T_{m, r}=T_{n, r} T_{m, r}^{*}=0, m \neq n$. Since $\|u \otimes v\|_{p}=\|u\|\|v\|$ for all $u, v \in H$, Lemma 4.2 and the hypothesis $(a)$ yield that $\sum_{n \leq r} R^{r^{q}-(r-n)^{q}}(x) \otimes\left(T^{*}\right)^{r^{q}-(r-n)^{q}}(y)$ is unconditionally convergent in $S_{p}(H)$, uniformly in $r$. Similarly, one can obtain that $\sum_{n} x_{k,(n+r)^{q}-r^{q}} \otimes$ $y_{k,(n+r)^{q}-r^{q}}$ is unconditionally convergent in $S_{p}(H)$, uniformly in $r$. Thus the condition $(a)$ of Theorem 3.3 is satisfied by $C_{R, T}$ in $S_{p}(H)$.

Next, from Theorem 4.3, we obtain conditions on the weight sequences $\left(w_{n}\right)$ and $\left(\mu_{n}\right)$ that are sufficient for the $C_{B_{w}, F_{\mu}}$ on $S_{p}\left(\ell^{2}\right)$ and $C_{T_{a}, S_{b}}$ on $S_{p}\left(\ell^{2}(\mathbb{Z})\right)$ to be $q$-frequently hypercyclic, $1 \leq p<\infty$. 
Proposition 4.4. (1) If $\sum_{n=1}^{\infty}\left|w_{1} w_{2} . . w_{(n+r)^{q}-r^{q}+i} \mu_{1} \mu_{2} . . \mu_{(n+r)^{q}-r^{q}+j}\right|^{-p}<\infty$ uniformly in $r \geq 0$ for all $i, j \geq 0$, then $C_{B_{w}, F_{\mu}}$ is q-frequently hypercyclic on $S_{p}\left(\ell^{2}\right)$.

(2) If $\sum_{n=1}^{\infty}\left|a_{1} a_{2} . . a_{(n+r)^{q}-r^{q}+i} b_{1} b_{2} . . b_{(n+r)^{q}-r^{q}+j}\right|^{-p}<\infty$ and

$\sum_{n=0}^{r}\left|a_{i} a_{i-1} . . a_{i-r^{q}+(r-n)^{q}+1} b_{j} b_{j-1} . . b_{j-r^{q}+(r-n)^{q}+1}\right|^{p}<\infty$ uniformly in $r \geq 0$ for all $i, j \in \mathbb{Z}$, then $C_{T_{a}, S_{b}}$ is q-frequently hypercyclic on $S_{p}\left(\ell^{2}(\mathbb{Z})\right)$.

Proof. To prove part (1), choose $D_{1}=D_{2}=\left\{e_{n}: n \geq 0\right\}$, the standard orthonormal basis in $\ell^{2}$. Let $S_{n}$ and $J_{n}$ be the maps as considered in the proof of Proposition 3.10, i.e., $S_{n}\left(e_{i}\right)=$ $S_{w}^{n}\left(e_{i}\right)=\frac{1}{w_{i+1 . . w_{i+n}}} e_{i+n}$ and $J_{n}\left(e_{j}\right)=J_{\mu}^{n}\left(e_{j}\right)=\frac{1}{\mu_{j+1} . \mu_{j+n}} e_{j+n}$. Note that $\left\langle S_{n}\left(e_{j}\right), S_{m}\left(e_{j}\right)\right\rangle=$ $\left\langle J_{n}\left(e_{j}\right), J_{m}\left(e_{j}\right)\right\rangle=0$ for $n \neq m$. As $B_{w}^{n}\left(e_{i}\right)=0$ for sufficiently large $n$, we have that

$\sum_{n \leq r}\left\|B_{w}^{r^{q}-(r-n)^{q}}\left(e_{i}\right)\right\|^{p}\left\|\left(F_{\mu}^{*}\right)^{r^{q}-(r-n)^{q}}\left(e_{j}\right)\right\|^{p}<\infty$ uniformly in $r$. Moreover, from the hypothesis, we have $\sum_{n}\left\|S_{(n+r)^{q}-r^{q}}\left(e_{i}\right)\right\|^{p}\left\|J_{(n+r)^{q}-r^{q}}\left(e_{j}\right)\right\|^{p}<\infty$ uniformly in $r$. Now Theorem 4.3 yields the result.

To prove part (2), consider the maps $S_{n}$ and $J_{n}$ in the proof of Proposition 3.11 and choose $D_{1}=D_{2}=\left\{e_{n}: n \in \mathbb{Z}\right\}$, the standard orthonormal basis in $\ell^{2}(\mathbb{Z})$. Then, for $i, j \in \mathbb{Z}$, we have

$$
S_{n}\left(e_{i}\right)=S^{n}\left(e_{i}\right)=\frac{1}{a_{i+1} a_{i+2} . . a_{i+n}} e_{i+n} \text { and } J_{n}\left(e_{j}\right)=J^{n}\left(e_{j}\right)=\frac{1}{b_{j+1} b_{j+2} . . b_{j+n}} e_{j+n},
$$

and

$$
T_{a}^{n}\left(e_{i}\right)=a_{i} a_{i-1} \ldots a_{i-n} e_{i-n-1} \text { and }\left(S_{b}^{*}\right)^{n}\left(e_{j}\right)=b_{j} b_{j-1} \ldots b_{j-n} e_{j-n-1} .
$$

It follows by the hypotheses that the series $\sum_{n \geq 1}\left\|S_{(n+r)^{q}-r^{q}}\left(e_{i}\right)\right\|^{p}\left\|J_{(n+r)^{q}-r^{q}}\left(e_{j}\right)\right\|^{p}$ as well as $\sum_{n \leq r}\left\|T_{a}^{r^{q}-(r-n)^{q}}\left(e_{i}\right)\right\|^{p}\left\|\left(S_{b}^{*}\right)^{r^{q}-(r-n)^{q}}\left(e_{j}\right)\right\|^{p}$ converges uniformly $r \geq 0$.

\section{Frequent Hypercyclicity in $S_{p}\left(H^{2}(\mathbb{D})\right)$ and $\mathcal{N}\left(\ell^{p}\right)$}

This section includes results on frequent hypercyclicity of specific operators of the form $C_{R, T}$ defined on the $p$ th Schatten von-Neumann class of operators on the Hardy space $H^{2}(\mathbb{D}), 1 \leq p<\infty$, as well as on the space $\mathcal{N}\left(\ell^{p}\right)$ of all nuclear operators on $\ell^{p}, 1<p<\infty$. Let us recall the Eigenvalue Criterion, due to S. Grivaux.

Proposition 5.1. [12] Let $X$ be a separable, complex Banach space and $T \in \mathcal{L}(X)$. If for every countable subset $D$ of the unit circle $S^{1}$, the set $\bigcup_{\alpha \in S^{1} \backslash D} \operatorname{Ker}(T-\alpha I)$ spans a dense subspace of $X$, then $T$ is frequently hypercyclic.

Corresponding to a sequence $\beta=\left(\beta_{n}\right), \beta_{n}>0, n \geq 0$, let $\left(H^{\beta}(\mathbb{D}),<.>\right)$ be a Hilbert space of complex functions, analytic on the open unit disc $\mathbb{D}$ such that the evaluation mappings $f \rightarrow f(z)$ are continuous at each $z \in \mathbb{D}$, i.e. there exists $k_{z} \in H^{\beta}(\mathbb{D})$ such that $f(z)=\left\langle f, k_{z}\right\rangle$ for each $f \in H^{\beta}(\mathbb{D})$. Such a function $k_{z}$ is called a reproducing kernel at $z \in \mathbb{D}$. Also, assume that $\left\{e_{n}\right\}_{n=0}^{\infty}$ forms an orthonormal basis for $H^{\beta}(\mathbb{D})$, where $e_{n}(z)=\beta_{n} z^{n}$. Note that when $\beta_{n}=1$ for all $n \geq 0$, we have the Hardy space $H^{2}(\mathbb{D})$. 
Let $M_{\varphi}$ be the multiplication operator $f(z) \rightarrow \varphi(z) f(z)$ on $H^{\beta}(\mathbb{D})$, corresponding to an analytic function $\varphi$ on $\mathbb{D}$ and $M_{\varphi}^{*}$ be the Hilbert space adjoint of $M_{\varphi}$. Our aim is to establish the frequent hypercyclicity of $C_{M_{\varphi}^{*}, M_{\psi}}$ on $S_{p}\left(H^{\beta}(\mathbb{D})\right)$, where $M_{\varphi}$ and $M_{\psi}$ are bounded multiplication operators on $H^{\beta}(\mathbb{D})$ corresponding to the analytic functions $\varphi$ and $\psi$ on $\mathbb{D}$. Let us first prove

Lemma 5.2. Let $\varphi$ and $\psi$ be non-zero analytic functions on $\mathbb{D}$ such that at least one of them is non-constant and $|\varphi(z) \psi(w)|=1$ for some $z, w \in \mathbb{D}$. Then

$$
\operatorname{span}\left\{k_{z} \otimes k_{w}: \overline{\varphi(z)} \psi(w) \in S^{1} \backslash D\right\}
$$

is dense in the space $S_{1}\left(H^{\beta}(\mathbb{D})\right)$ for every countable set $D \subset S^{1}$.

Proof. We write $\mathcal{H}=H^{\beta}(\mathbb{D})$ and $\overline{\varphi(\mathbb{D})}=\{\overline{\varphi(z)}: z \in \mathbb{D}\}$. By the open mapping theorem for analytic functions, the set $\overline{\varphi(\mathbb{D})} \psi(\mathbb{D})=\{\overline{\varphi(z)} \psi(w): z, w \in \mathbb{D}\}=\bigcup_{z \in \mathbb{D}}(\overline{\varphi(z)} \psi(\mathbb{D}))$ is non-empty and open. Hence there exists an open arc $\Gamma$ in $S^{1}$ such that $\Gamma \subset \overline{\varphi(\mathbb{D})} \psi(\mathbb{D})$; let us assume that this arc $\Gamma$ is the maximal one.

Consider the set

$$
U \times V=\{(z, w) \in \mathbb{D} \times \mathbb{D}: \overline{\varphi(z)} \psi(w) \in \Gamma \backslash D\} .
$$

We claim that $U$ is uncountable, and for each $z \in U$, there exists an uncountable set $V_{1} \subseteq V$ such that

$$
\overline{\varphi(z)} \psi(w) \in \Gamma \backslash D, \text { for all } w \in V_{1} .
$$

To prove this, assume that both $\varphi$ and $\psi$ are non-constant. In this case, $\overline{\varphi(\mathbb{D})}$ is a non-empty open set. If $|\overline{\phi(z)} \psi(w)|=1$ for some $(z, w) \in \mathbb{D} \times \mathbb{D}$, then $\psi(w) \overline{\varphi(\mathbb{D})}$ is non-empty and open and so, we can find an arc $\Gamma_{1} \subset \Gamma$ such that $\Gamma_{1} \subset \psi(w) \overline{\varphi(\mathbb{D})}$ as above. Since $D$ is countable and $\Gamma_{1} \backslash D \subset \psi(w) \overline{\varphi(\mathbb{D})}$, the set $U$ has to be uncountable. Now fix $z \in U$. Then the set $\overline{\varphi(z)} \psi(\mathbb{D})$, being non-empty and open, contains $\Gamma_{2} \backslash D$ for some sub-arc $\Gamma_{2}$ of $\Gamma$. This proves the claim when $\varphi$ and $\psi$ are non-constant. Now assume that $\psi$ is constant, say $\psi=c$ and $\varphi$ is non-constant. We can proceed as above to prove that the set $U$ is uncountable since $\overline{c \varphi(\mathbb{D})}$ is a non-empty open set containing $\Gamma \backslash D$. Also, since $\psi$ is constant, we can take $V_{1}=V=\mathbb{D}$. Finally when $\varphi$ is constant and $\psi$ is non-constant, we proceed similarly to get the result. Hence our claim is established.

We now show that $\Lambda=\operatorname{span}\left\{k_{z} \otimes k_{w}: z \in U, w \in V\right\}$ is dense in the space $S_{1}(H)$. Recall that the trace of $A \in S_{1}(\mathcal{H})$ is given by $\operatorname{tr}(A)=\sum_{n \geq 0}\left\langle A e_{n}, e_{n}\right\rangle$, where $e_{n}(z)=\beta_{n} z^{n}, n \geq 0$. Also we have $S_{1}(H)^{*}=\mathcal{L}(H)$ with respect to the duality-pairing $(A, T)=\operatorname{tr}(A T), T \in S_{1}(H)$ and $A \in \mathcal{L}(H)$.

Let $A \in \mathcal{L}(H)$ be such that $\operatorname{tr}(A T)=0$ for all $T \in \Lambda$. For $T=k_{z} \otimes k_{w}$, we have $T e_{n}=\left\langle e_{n}, k_{w}\right\rangle k_{z}=$ 
$e_{n}(w) k_{z}=\beta_{n} w^{n} k_{z}$ and

$$
\begin{aligned}
\operatorname{tr}(A T) & =\sum_{n \geq 0}\left\langle A T e_{n}, e_{n}\right\rangle \\
& =\sum_{n \geq 0} \beta_{n} w^{n}\left\langle k_{z}, A^{*} e_{n}\right\rangle \\
& =\sum_{n \geq 0} \beta_{n} \overline{\left(A^{*} e_{n}\right)(z)} w^{n} .
\end{aligned}
$$

Since $A T \in S_{1}(H)$, the above power series is well-defined for all $z, w \in \mathbb{D}$. Hence it is an analytic function in the variable $w$ for a fixed $z \in \mathbb{D}$. For $z \in U$, there exists an uncountable set $V_{1}$ such that (5.1) holds. Since $V_{1}$ is uncountable, it has a limit point in $\mathbb{D}$. As $\beta_{n}>0$ for all $n \geq 0$ and $\operatorname{tr}(A T)=0$, it follows that the coefficients of the above power series are all zero, i.e., $A^{*}\left(e_{n}\right)(z)=0$ for all $n \geq 0$. Similarly, since $z \in U$ is arbitrary and $U$ is uncountable, we have $A^{*}\left(e_{n}\right)=0, \forall n \geq 0$. As $\left\{e_{n}: n \geq 0\right\}$ spans a dense subspace of $\mathcal{H}$, we conclude that $A=0$. Therefore the set $\Lambda$ is dense in $S_{1}(\mathcal{H})$. The proof is now complete.

The above lemma yields

Theorem 5.3. Let $\varphi$ and $\psi$ be non-zero analytic functions on $\mathbb{D}$ such that the corresponding multiplication operators are bounded on $H^{\beta}(\mathbb{D})$. If one of the maps $\varphi$ and $\psi$ is non-constant and $|\varphi(z) \psi(w)|=1$ for some $z, w \in \mathbb{D}$, then $C_{M_{\varphi}^{*}, M_{\psi}}$ is frequently hypercyclic on $\left(S_{p}\left(H^{\beta}(\mathbb{D})\right),\|\cdot\|_{p}\right)$, $\left(\mathcal{K}\left(H^{\beta}(\mathbb{D})\right),\|\cdot\|_{\text {op }}\right)$, and $\left(\left(\mathcal{L}\left(H^{\beta}(\mathbb{D})\right), C O T\right)\right)$.

Proof. For $z, w \in \mathbb{D}$, consider $k_{z} \otimes k_{w} \in S_{1}(H)$. Since $M_{\varphi}^{*}\left(k_{z}\right)=\overline{\varphi(z)} k_{z}$, we have

$$
C_{M_{\varphi}^{*}, M_{\psi}}\left(k_{z} \otimes k_{w}\right)=\overline{\varphi(z)} \psi(w)\left(k_{z} \otimes k_{w}\right)
$$

Thus $k_{z} \otimes k_{w}$ is an eigen vector for $C_{M_{\varphi}^{*}, M_{\psi}}$ corresponding to the eigen value $\overline{\varphi(z)} \psi(w)$. Now by Lemma [5.2, $\operatorname{span}\left\{k_{z} \otimes k_{w}: \overline{\varphi(z)} \psi(w) \in S^{1} \backslash D\right\}$ is dense in $S_{1}\left(H^{\beta}(\mathbb{D})\right)$ for any countable set $D \subset S^{1}$. Hence $C_{M_{\varphi}^{*}, M_{\psi}}$ is frequently hypercyclic on $\left(S_{1}\left(H^{\beta}(\mathbb{D})\right),\|\cdot\|_{1}\right)$ by Proposition [5.1. Since the embeddings $S_{1}\left(H^{\beta}(\mathbb{D})\right) \hookrightarrow S_{p}\left(H^{\beta}(\mathbb{D})\right) \hookrightarrow \mathcal{K}\left(H^{\beta}(\mathbb{D})\right) \hookrightarrow\left(\mathcal{L}\left(H^{\beta}(\mathbb{D})\right), C O T\right)$ are continuous and have dense range, it follows that $C_{M_{\varphi}^{*}, M_{\psi}}$ is frequently hypercyclic on each of the these spaces.

As noted in the beginning of this section, the Hardy space $H^{2}(\mathbb{D})$ is a special case of $H^{\beta}(\mathbb{D})$. In this case, we have the following characterization for $C_{M_{\varphi}^{*}, M_{\psi}}$ on $S_{p}\left(H^{2}(\mathbb{D})\right)$ and $\mathcal{K}\left(H^{2}(\mathbb{D})\right)$.

Theorem 5.4. Let $\varphi$ and $\psi$ be non-zero, bounded and analytic on $\mathbb{D}$, with one of them being non-constant. Then $C_{M_{\varphi}^{*}, M_{\psi}}$ is frequently hypercyclic on $\left(S_{p}\left(H^{2}(\mathbb{D})\right),\|\cdot\|_{p}\right),\left(\mathcal{K}\left(H^{2}(\mathbb{D})\right),\|\cdot\|_{o p}\right)$ and $\left(\mathcal{L}\left(H^{2}(\mathbb{D})\right), C O T\right)$ if $|\varphi(z) \psi(w)|=1$ for some $z, w \in \mathbb{D}$. Conversely, if $C_{M_{\varphi}^{*}, M_{\psi}}$ is frequently hypercyclic on $\left(\mathcal{K}\left(H^{2}(\mathbb{D})\right),\|\cdot\|_{o p}\right)$ or $\left(S_{p}\left(H^{2}(\mathbb{D})\right),\|\cdot\|_{p}\right)$, then $|\varphi(z) \psi(w)|=1$ for some $z, w \in \mathbb{D}$.

Proof. We know that $M_{\varphi} \in \mathcal{L}\left(H^{2}(\mathbb{D})\right)$ if and only if $\varphi$ is bounded on $\mathbb{D}$. Thus by the preceding theorem, if $|\varphi(z) \psi(w)|=1$ for some $z, w \in \mathbb{D}$, then $C_{M_{\varphi}^{*}, M_{\psi}}$ is frequently hypercyclic. 
Let $G=\varphi(\mathbb{D}) \psi(\mathbb{D})$. Assume that the converse is not true, i.e., $G \cap S^{1}=\phi$. Then $G \subseteq \mathbb{D}$ or $G \subseteq \mathbb{C} \backslash \overline{\mathbb{D}}$ since $G$ is non-empty and open, where $\overline{\mathbb{D}}$ is the closed unit disc in $\mathbb{C}$. In case $G \subseteq \mathbb{D}$, then $\|\varphi\|_{\infty}\|\psi\|_{\infty}=\sup _{z \in \mathbb{D}}|\varphi(z)| \sup _{w \in \mathbb{D}}|\psi(w)|=\sup _{z, w \in \mathbb{D}}|\varphi(z) \psi(w)| \leq 1$, and consequently,

$$
\left\|C_{M_{\varphi}^{*}, M_{\psi}}\right\| \leq\left\|M_{\varphi}^{*}\right\|\left\|M_{\psi}\right\| \leq\|\varphi\|_{\infty}\|\psi\|_{\infty} \leq 1 .
$$

In the latter case when $G \subseteq \mathbb{C} \backslash \overline{\mathbb{D}}$, we have $\inf _{z, w \in \mathbb{D}}|\varphi(z) \psi(w)| \geq 1$, and so, $\inf _{z \in \mathbb{D}}|\varphi(z)|>0$ and $\inf _{w \in \mathbb{D}}|\psi(w)|>0$. Thus the functions $\varphi^{-1}$ and $\psi^{-1}$ are bounded and analytic on $\mathbb{D}$, and the corresponding operators $M_{\varphi^{-1}}, M_{\psi^{-1}}$ are bounded on $H^{2}(\mathbb{D})$. Moreover $\left(M_{\varphi}\right)^{-1}=M_{\varphi^{-1}}$ and $\left(M_{\varphi}^{*}\right)^{-1}=M_{\varphi^{-1}}^{*}$. Then we observe that

$$
\left\|\left(C_{M_{\varphi}^{*}, M_{\psi}}\right)^{-1}\right\|=\left\|C_{M_{\varphi^{-1}}^{*}, M_{\psi^{-1}}}\right\| \leq\left\|M_{\varphi^{-1}}^{*}\right\|\left\|M_{\psi^{-1}}\right\| \leq\left\|\varphi^{-1}\right\|_{\infty}\left\|\psi^{-1}\right\|_{\infty} \leq 1 .
$$

Thus, in both the cases, the operator $C_{M_{\varphi}^{*}, M_{\psi}}$ is not hypercyclic on $\mathcal{K}\left(H^{2}(\mathbb{D})\right)$. This contradiction proves that $G \cap S^{1} \neq \phi$.

As a special case, we state below the frequent hypercyclicity of the conjugate operator $C_{M_{\varphi}^{*}}$ for the Hardy space $H=H^{2}(\mathbb{D})$.

Proposition 5.5. If $\varphi$ is non-constant and $|\varphi(z)|=1$ for some $z \in \mathbb{D}$, then the conjugate map $C_{M_{\varphi}^{*}}$ is frequently hypercyclic on $\left(S_{p}(H),\|\cdot\|_{p}\right),\left(\mathcal{K}(H),\|\cdot\|_{o p}\right)$ and $(\mathcal{L}(H), C O T)$. Conversely, if $C_{M_{\varphi}^{*}}$ is frequently hypercyclic on $\left(\mathcal{K}(H),\|\cdot\|_{o p}\right)$, then $\varphi$ is non-constant and $\varphi(\mathbb{D}) \cap S^{1} \neq \phi$.

Proof. Immediate from the preceding theorem.

The above characterization is not true for multiplication operators on all Hilbert spaces of analytic functions, e.g. consider

Example 5.6. Let $\mathcal{H}=\left\{f(z)=\sum_{n \geq 0} a_{n} z^{n}:\|f\|^{2}=\sum_{n \geq 0}(n+1)^{2}\left|a_{n}\right|^{2}<\infty\right\}$. Then $\mathcal{H}$ is a reproducing kernel Hilbert space and the multiplication operator $M_{\varphi}$ corresponding to $\varphi(z)=z$ acts as the shift

$$
M_{\varphi}\left(e_{n}\right)(z)=z e_{n}(z)=\frac{1}{n+1} z^{n+1}=\frac{n+2}{n+1} e_{n+1}(z)
$$

with respect to the orthonormal basis $e_{n}(z)=\frac{1}{n+1} z^{n}, n \geq 0$. Now $w_{1} w_{2} \ldots w_{n}=n+1$ implies that $\sum_{n} \frac{1}{\left(w_{1} w_{2} . . w_{n}\right)^{2}}<\infty$ and consequently, the adjoint $M_{\varphi}^{*}$ satisfies the FHC Criterion on $\mathcal{H}$. Hence by Corollary [3.9, $C_{M_{\varphi}^{*}}$ is frequently hypercyclic on the spaces $\left(S_{p}(H),\|\cdot\|_{p}\right),\left(\mathcal{K}(\mathcal{H}),\|\cdot\|_{o p}\right)$ and $(\mathcal{L}(\mathcal{H}), C O T)$. However there are no $z, w \in \mathbb{D}$ such that $|\varphi(z) \varphi(w)|=1$.

In the spirit of Theorem 5.3, let us prove a similar result about $C_{\phi(B), \psi(F)}$ defined on spaces of operators on $\ell^{p}, 1<p<\infty$, where $\varphi(B)$ and $\varphi(F)$ are functions of the unweighted backward and forward shifts respectively. If $\varphi(z)=\sum_{n \geq 0} a_{n} z^{n}$ is an analytic function on some neighborhood of the closed disc $\overline{\mathbb{D}}$, then $\varphi(B)=\sum_{n} a_{n} B^{n}$ and $\varphi(F)=\sum_{n} a_{n} F^{n}$ are bounded operators on $\ell^{p}$, $1 \leq p<\infty$. Moreover, the Banach space adjoint of $\varphi(B)$ is $\phi(F)$ and that of $\varphi(F)$ is $\varphi(B)$. Note that if $f_{\lambda}=\left(1, \lambda, \lambda^{2}, \ldots\right)$ and $|\lambda|<1$, then $\varphi(B) f_{\lambda}=\varphi(\lambda) f_{\lambda}$. In [9], R. Delaubenfels and H. Emamirad proved that if $\varphi(\mathbb{D}) \cap S^{1} \neq \phi$, then $\varphi(B)$ is hypercyclic on $\ell^{p}$. We now have the following result, which can be proved using Proposition 5.1, 
Proposition 5.7. If $\varphi$ is non-constant and $\varphi(\mathbb{D}) \cap S^{1} \neq \phi$, then $\varphi(B)$ is frequently hypercyclic on $\ell^{p}, 1 \leq p<\infty$.

Let $\mathcal{N}\left(\ell^{p}\right)$ denote the space of all nuclear operators on $\ell^{p}$. Then the trace $\operatorname{tr}(T)=\sum_{n} x_{n}^{*}\left(x_{n}\right)$ of $T=\sum_{n} x_{n} \otimes x_{n}^{*} \in \mathcal{N}\left(\ell^{p}\right), 1<p<\infty$. Then the dual of $\mathcal{N}\left(\ell^{p}\right)$ is identified with $\mathcal{L}\left(\ell^{p}\right)$ via the trace-duality $(S, T)=\operatorname{tr}(T S)$, where $T \in \mathcal{N}\left(\ell^{p}\right)$ and $S \in \mathcal{L}\left(\ell^{p}\right)$, cf. [11], Theorem 16.50. The one-rank operator $f_{\lambda} \otimes f_{\mu}$ on $\ell^{p}$ is given by $x \rightarrow f_{\mu}(x) f_{\lambda}$.

Lemma 5.8. Let $\varphi$ and $\psi$ be non-zero functions analytic on some neighborhoods of the closed disc $\overline{\mathbb{D}}$ with one of them being non-constant and $|\varphi(\lambda) \psi(\mu)|=1$ for some $\lambda, \mu \in \mathbb{D}$. Then

$$
\operatorname{span}\left\{f_{\lambda} \otimes f_{\mu}: \varphi(\lambda) \psi(\mu) \in S^{1} \backslash D\right\}
$$

is dense in $\mathcal{N}\left(\ell^{p}\right)$ for every countable set $D \subset S^{1}$ and $1<p<\infty$.

Proof. Invoking the proof of Lemma 5.2, we can find an arc $\Gamma \subseteq S^{1} \cap \varphi(\mathbb{D}) \psi(\mathbb{D})$. Write $U \times V=$ $\{(\lambda, \mu) \in \mathbb{D} \times \mathbb{D}: \varphi(\lambda) \psi(\mu) \in \Gamma \backslash D\}$. Then, for each $\mu \in V$, there exists an uncountable set $U_{1} \subset \mathbb{D}$ such that for $\lambda \in U_{1}$ we have $\varphi(\lambda) \psi(\mu) \in \Gamma \backslash D$.

Let us now prove that $\Delta=\operatorname{span}\left\{f_{\lambda} \otimes f_{\mu}: \lambda \in U, \mu \in V\right\}$ is dense in $\mathcal{N}\left(\ell^{p}\right)$. For this, let $S \in \mathcal{L}\left(\ell^{p}\right)$ such that $\operatorname{tr}(T S)=0$ for all $T \in \Delta$. In particular, if $T=f_{\lambda} \otimes f_{\mu}$ for $\lambda \in U_{1}$, then $\operatorname{tr}\left(f_{\lambda} \otimes S^{*} f_{\mu}\right)=\left(S^{*} f_{\mu}\right)\left(f_{\lambda}\right)=0$. Since $U_{1}$ has limit points in $\mathbb{D}$, we have that $\operatorname{span}\left\{f_{\lambda}: \lambda \in U_{1}\right\}$ is dense in $\ell^{p}$. Thus $S^{*}\left(f_{\mu}\right)=0$ for all $\mu \in V$. As $\operatorname{span}\left\{f_{\lambda}: \mu \in V\right\}$ is dense in $\ell^{p^{*}}$, the dual of $\ell^{p}$, $S=0$.

Using the above lemma, we prove the frequent hypercyclicity of $C_{\varphi(B), \psi(F)}$ as follows.

Theorem 5.9. Suppose $\phi$ and $\psi$ are non-zero analytic maps on some neighborhoods of the closed disc $\overline{\mathbb{D}}$ such that $|\phi(z) \psi(w)|=1$ for some $z, w \in \mathbb{D}$, with one of $\varphi$ and $\psi$ being non-constant. Then $C_{\phi(B), \psi(F)}$ is frequently hypercyclic on $\left(\mathcal{N}\left(\ell^{p}\right),\|\cdot\|_{n u}\right),\left(\mathcal{K}\left(\ell^{p}\right),\|\cdot\|_{o p}\right)$ and $\left(\mathcal{L}\left(\ell^{p}\right), C O T\right)$ for $1<p<\infty$.

Proof. Let $\lambda, \mu \in \mathbb{D}$. Since $\phi(B)\left(f_{\lambda}\right)=\phi(\lambda) f_{\lambda}$, we get

$$
C_{\phi(B), \psi(F)}\left(f_{\lambda} \otimes f_{\mu}\right)=\varphi(B)\left(f_{\lambda}\right) \otimes \psi(B)\left(f_{\mu}\right)=\phi(\lambda) \psi(\mu)\left(f_{\lambda} \otimes f_{\mu}\right),
$$

where $f_{\lambda}=\left(1, \lambda, \lambda^{2}, \ldots\right)$. From Lemma [5.8, it follows that span $\left\{f_{\lambda} \otimes f_{\mu}: \varphi(\lambda) \psi(\mu) \in S^{1} \backslash D\right\}$ is dense in $\mathcal{N}\left(\ell^{p}\right)$ and the proof is complete by Proposition 5.1 .

Acknowledgements. The second author acknowledges the Council of Scientific and Industrial Research INDIA for a research fellowship.

\section{REFERENCES}

[1] Bayart, F.; Grivaux, S.: Frequently hypercyclic operators Trans. Amer. Math. Soc. 358(2006), 5083-5117.

[2] Bayart, F.; Matheron, E.: Dynamics of Linear Operators Cambridge Univ. Press 179(2009). 
[3] Bonet, J., Martinez-Gimenez, F., Peris, A.: Universal and chaotic multipliers on spaces of operators J. Math. Anal. Appl 297 (2004) no. 2, 599-611.

[4] Bonilla, A.; Grosse-Erdmann, K-G.: Frequently hypercyclic operators and vectors, Ergodic Theory Dynam. Systems 27(2007), 383-404. Erratum: Ergodic Theory Dynam. Systems 29 (2009), 1993-1994.

[5] Bonilla, A., Grosse-Erdmann, K-G.: Frequently hypercyclic subspaces Monatsh. Math. 168 (2012) no. 3-4, 305-320.

[6] Carbery, A.: Almost-orthogonality in the Schatten-von Neumann classes J. Operator Theory. 62 (2009), no.1, 151-158.

[7] Chan, K.: Hypercyclicity of the operator algebra for a separable Hilbert space J. Operator Theory 42 (1999) no. 2, 231-244.

[8] Chan, K.; Taylor, R.: Hypercyclic subspaces of a Banach space, Integral Equations Operator Theory 41 (2001), no. 4, 381-388.

[9] Delaubenfels, R.; Emamirad, H.: Chaos for functions of discrete and continuous weighted shift operators Ergodic theory and Dynamical Systems 21, 1411-1427 (2001).

[10] Diestal, J.; Jarcho, H.; Tonge, A.: Absolutely Summing Operators Vol. 43, Cambridge Studies in Advanced Mathematics, Cambridge Univ. Press, 1995.

[11] Fabian, M.; Habala, P.; Hajek, P.; Montesinos, V.; Zizler, V.: Banach Space Theory: The Basis for Linear and Non-linear Analysis Springer, New York, 2011.

[12] Grivaux, S.: A new class of frequently hypercyclic operators with applications Indiana Univ. Math. J. 60 (2011), no. 4, 1177-1201.

[13] Grosse-Erdmann, K-G.; Peris, A.: Linear Chaos Universitext. Springer, London, 2011.

[14] Gupta,, M.; Mundayadan, A.: q-Frequently hypercyclic operators Banach J. Math. Anal., vol. 9, no. 2 (2015) 114-126.

[15] Kamthan, P.K; Gupta, M.: Sequence Spaces and Series, Marcell-Dekker, 65 (1981).

[16] Lindenstrauss, J.; Tzaffiri, T.: Classical Banach Spaces Springer-Verlag, 1997.

[17] Martinez-Gimenez, F.; Peris, A.: Universality and chaos for tensor produtcs of operators J. Approx. Theory 124 (2003) no.1, 7-24.

[18] McArthur, C.W.; Retherford, J.R.: Some applications of an inequality in locally convex spaces Trans. Amer. Math. Soc., 137 (1969), 115-123.

[19] Petersson, H.: Hypercyclic conjugate operators Integral Equations Operator Theory 57 (2007), 413-423.

Department of Mathematics and Statistics, Indian Institute of Technology Kanpur, 208016 KanPUR, INDIA

E-mail address: manjul@iitk.ac.in

Department of Mathematics and Statistics, Indian Institute of Technology Kanpur, 208016 KanPUR, INDIA

E-mail address: aneeshm@iitk.ac.in 